NEUROPSYCHOLOGIE CLINIQUE ET NEUROLOGIE DU COMPORTEMENT. 1996. Edited by Mihai Ioan Botez. Published by Gaetan Morin. 682 pages. $\$ C 68.00$

Unquestionably, we are in the midst of an unparalleled explosion of information in cognitive neuroscience, extending from knowledge of the molecular basis of learning, to new treatments for Alzheimer's Disease, to a new functional neuroanatomy based on PET activation studies of normal subjects. It is no longer quite clear what portion of this data explosion ought to be distilled into a book written for clinical neuropsychologists and behavioral neurologists. This is the second edition of Dr. Mihai Botez's popular clinical text, in which he deals reasonably successfully with this difficult question of subject matter. Drawing on thirty years' work in behavioral neurology at the Université de Montréal, Dr. Botez has brought together experts from North America, England, and France to produce a dense text which should satisfy the clinical needs of most students of neuropsychology.

Dr. Botez's book begins with a historical introduction to the issues of localization of cognitive function, as well as a clear briefing on the anatomy of cognition. Six chapters then deal with various methods of investigation now active in neuropsychology - the neuropsychological evaluation, information processing systems and their investigation with reaction time measures, electrophysiology (especially evoked potentials), brain imaging with CT, MRI, SPECT, and PET, and neurochemical indices of behavior. Further chapters deal with the traditional domains of neuropsychology. Localized syndromes are discussed in terms of frontal, parietal, temporal, occipital regions, along with syndromes ascribable to basal ganglia, thalamic, and cerebellar disease. Issues of functional assymetry of the brain and callosal function are also dealt with in separate chapters. The next section deals with the "grand syndromes" in clinical neuropsychology and behavioral neurology - amnesia, apraxia, agnosia, aphasia, and alexia. Next, chapters deal with attentional impairments from Attention Deficit Disorder and head trauma, along with a set of chapters on normal aging and the various dementias.

This is a thoughtful and well-prepared book which should be on the bookshelf of anybody interested in disorders of cognition who is fluent in French. The enormous complexity of coordinating 55 experts in producing a book of uniform high quality is testament to the dedication of Dr. Botez to the field. It is a pleasure to read Dr. Botez's chapter on the motor and non-motor role of the cerebellum; 27 years after he first claimed a role for the cerebellum in cognition, and 13 years after his first clear data on the subject, the rest of the cognitive neuroscience community has swung over to this view in the wake of undeniable imaging and lesion data. Dr. Botez's contrition in this area is, with hindsight, undeniable.

This is a book which is enviable in its breadth. Dr. Botez has included very readable chapters covering the neuropsychology of Sleep Apnea Syndrome, epilepsy, and the amusias. There are chapters dealing in detail with emotions and behavior, as well as hallucinations and illusions. Each chapter is densely written - the aphasia chapter by Drs. Verstichel and Cambier, for instance, covers most of the material included in recent books on the subject!

In contrast, there are certain areas where one wishes there were fewer authors and more cohesion, or else fewer chapters and more detail. None of the "grand syndromes" chapters adequately incorporate recent discoveries from brain imaging studies, for instance. Discussion of such functional studies is either absent (in the case of reading) or else summarized in two pages in a chapter on PET earlier on.

This book suffers from gaps, to be sure; given the knowledge explosion, there is no choice but to condense and omit. Some of the gaps are somewhat surprising. Behavioral neurology is currently being rapidly transformed from an arcane discipline concerned with descriptions of interesting but untreatable cognitive syndromes, to a neurological subspecialty with legitimate concerns about accurate diagnosis and evidence-based evaluation of alternatives in treatment. The reader hoping to gain expertise in this growing medical side of behavioral neurology will be frustrated by Botez's book. Here the reader will find the clinical evaluation of Normal Pressure Hydrocephalus discussed in half a page, with another half page devoted to all aspects of its treatment. The growing focus on the "non-Alzheimer's Disease degenerative dementias" - Picks Disease, Lewy Body Dementia, Primary Progressive Aphasia receives all of less than three pages out of 667 . All aspects of the treatment of Alzheimer's Disease are covered in three pages.

On the other hand, there are numerous "gems" which are surprisingly concise and illuminating. Three chapters deal with recovery from cognitive disability in all of its aspects. Barbara Wilson has written an excellent chapter on Cognitive Rehabilitation, which is perhaps the best summary of the field one can find. The chapter on Legal aspects of neuropsychological evaluation provides a unique overview of the complex issues involved in neuropsychology "expertise" evaluations, which are a growing concern in the field. Denis Phaneuf and Thérèse Botez-Marquard have written an excellent summary chapter on Chronic Fatigue Syndrome which is the first I have seen in a Behavioral Neurology text (but I doubt it will be the last!). As these patients become more common (or at least the diagnosis becomes more common), familiarity with this syndrome will become a necessary part of neurology training.

I would recommend this book to anyone interested in the details of behavioral neurology and cognitive syndromes. It will remain the standard French reference book on the subject for years to come.

Howard Chertkow Montreal, Quebec

\section{CENTRAL NERVOUS SYSTEM INFECTIOUS DISEASES AND THERAPY. 1997. Edited by Karen L. Roos. Published by Marcel Dekker. 784 pages. $\$ C 254.00$}

In the current era of emerging infectious diseases, infections of the central nervous system (CNS) and associated complications represent some of the most devastating consequences of the new epidemics. The prime example is the AIDS pandemic in which $80 \%$ of people infected with human immunodeficiency virus type-1 (HIV-1) experience some form of neurological illness during the course of infection. At the same time, there has been a resurgence of previously recognized CNS infections including tuberculosis and malaria. In addition, improved survival following transplantation or treatment of malignancies has increased the number of patients who are susceptible to CNS infections. New technologies including refined neuroimaging and molecular detection methods have made it easier to diagnose and manage patients with infectious CNS diseases. Moreover, with the plethora of new drugs and increasing patterns of resistance to many established drugs, treatment of CNS infections and their complications has become more complex. Thus, a monograph providing both clinical descriptions and treatment of CNS infections is timely.

Central Nervous System Infectious Diseases and Therapy, edited by Karen Roos, is a comprehensive textbook on this subject including sections devoted to pediatric and adult infections. The editor has 
selected multiple authors from different disciplines, many of whom are internationally respected specialists. Each chapter covers a specific infection and addresses clinical manifestations, diagnosis, pathophysiology, and treatment. The areas dealt with in the text include infections encountered in both first world and developing countries. Several chapters deal with topics not usually covered in textbooks of neurological infections including facial palsy, GuillainBarre syndrome, the utility of specific diagnostic tools including PCR, neurological complications of chemotherapy for infections, and acute management of neurological infections.

Most of the chapters are clearly written and well referenced, providing the reader with current reviews of rapidly evolving areas. For example, the chapters on bacterial meningitis, Lyme Disease, HTLV-1, fungal infections, AIDS-related infections, and CSF shunt infections give the reader a systematic approach to each topic. Each chapter varies widely in the extent to which pathophysiology is discussed. Herein lies the major shortcoming of the text; there is tremendous variability between chapters. Several chapters are lacking in sufficient references or overlook important references; notably, the chapters on subdural emphyema, epidural abscess, and viral encephalitis are surprisingly brief. In contrast, the chapter on rabies provides a lengthy review of the subject with a exhaustive bibliography but misses some important references. Another striking gap is in the chapter on spongiform encephalopathies. Although there is some description of the colorful characters in this tempestuous field and a very clear explanation of the pathogenesis of prion protein-related disorders, the new variant of Creutzfeld-Jakob receives scant attention. Despite the breadth and relative flux of the entire subject of CNS infections, all of the authors have attempted to critically assess the literature, pertaining to treatment.

I am pleased to recommend this book. The clinical focus of this text makes it an indepth and useful reference to neurologists, neurosurgeons, infectious disease, and intensive care specialists. Not only will the clinical descriptions be helpful to trainees but it will keep practioners abreast of the new developments in diagnostic tools and treatments of CNS infections.

Christopher Power Winnipeg, Manitoba

PERSPECTIVES OF MOTOR BEHAVIOR AND ITS NEURAL BASIS. 1997. Edited by M.-C. Hepp-Reymond and G. Marini. Published by Karger. 138 pages. $\$ \mathrm{Cl} 107.00$

This book arose from a symposium held at the University of Fribourg in Switzerland in late 1994, in honor of Mario Wiesendanger's anticipated retirement as Chair of Physiology and Director of the Institute of Neurophysiology in Fribourg. Mario Wiesendanger made tremendous contributions to motor physiology with studies in both humans and primates; it is therefore quite fitting to have this book dedicated to him on the occasion of his retirement.

The book consists of 10 chapters from authors in Switzerland, France and Italy. The first chapter by Hepp-Reymond gives an elegant historical account of the investigations of the pyramidal tract, with emphasis on Wiesendanger's research and his views. This is followed by two chapters on grasping. Jeannerod discusses the contributions of anticipatory mechanisms and reflex adjustments to grasping in humans. The chapter by Rouiller and colleagues describes how the inactivation of the simian motor cortex by lidocaine injection affects the precision grip, while inactivation of the supplementary motor area (SMA) does not. They suggest that preci- sion grip movements are overtrained, and suggest that the SMA may be more involved in learning or the execution of more complex movements. The chapter by Massion discusses the central mechanisms for control of balance during movement. Pedotti describes a system for analysis of movements in 3D, and Dietz discusses his work on locomotor training using a treadmill in paraplegic patients. Marini eloquently describes the physiology of normal motor phenomena of sleep, including changes in cortical excitability during NREM and REM sleep, and atonia of REM sleep. However, the section on motor disorders during sleep is disappointing, since there was little discussion on the pathophysiology of these disorders. Buser and colleagues provide interesting data on subdural recordings of slow cortical potentials preceding movements. Of particular interest is the contingent negative variation (CNV), a slow scalp negative potential occurring between a warning signal and a go signal in a reaction time paradigm, which is thought to represent motor preparation. While scalp recorded CNV show wide distribution, subdural recordings show that it arises from the contralateral primary motor cortex, contralateral premotor cortex and bilateral SMA. Hess and his co-authors give an useful account of the use of transcranial magnetic stimulation (TMS) in the study of motor system. However, in their discussion of the site of stimulation of TMS they fail to mention the work of Edgley which showed that TMS can activate corticospinal neurons directly, in addition to activating them via interneurons. They ascribe inhibition of motor response as a result of prior stimulation of the contralateral hemisphere to trancallosal inhibition without providing any evidence. I find the last chapter by Mario Wiesendanger most interesting. He discusses the evolution of motor control research from the traditions of physiology, neurology and psychology to the present day integration of three disciplines. Not only does he give an interesting historical account, he also illustrates how many of these findings are still highly relevant to motor control research today.

Overall, the book is well written and provides useful information for anyone interested in motor control. There are however a number of shortcomings. It is neither comprehensive nor authoritative. There is no central theme, but rather a collection of the topics that reflect the author's research interest. Although published in 1997, it appears that most of the chapters are written in 1994 or 1995. The high price for this small book makes it more appropriate for purchase by libraries rather than individuals. Nevertheless, it is a useful reference for neuroscientists and clinicians interested in motor control.

Robert Chen Bethesda, Maryland

DRUG-INDUCED NEUROLOGICAL DISORDERS. 1996. By K.K. Jain. Published by Hogrefe \& Huber Publishers. 389 pages. $\$ C 127.00$

In an environment of increasing polypharmacy, drug toxicity must be considered in virtually every differential diagnosis. Physicians rapidly come to realize that the CPS (or PDR, for our US colleagues) is neither complete nor reliable, in addition to be lacking in specific references. In the CPS, peripheral neuropathy or at least "numbness" are listed under virtually every medication. Further, this is largely fossilized data reflecting clinical trial adverse drug reactions, which may not capture some rare but significant toxic disorders; these only become apparent when the medications are more widely used in the clinical setting. 